\title{
A CMOS optical sensor which counts the number of objects
}

\author{
Member Masatoshi Nishimura (Sankyo Co., Ltd.) \\ Non-member Jan Van der Spiegel (University of Pennsylvania)
}

\begin{abstract}
A CMOS optical sensor which measures the number and the size of objects has been designed and successfully tested. The sensor contains an array of $24 \times 14$ pixels in a chip area of $6.8 \mathrm{~mm} \times 4.6 \mathrm{~mm}$. The sensor first performs image digitization by comparing a photogenerated current with a given threshold. A Winner-Take-All (WTA) operation is carried out next in each object region which is defined as a collection of illuminated pixels mutually connected in either a horizontal or a vertical direction. Realization of the WTA circuit with sharp selectivity makes it possible to select only one winner from each object by utilizing inherent mismatch in transistor characteristics. The output current of the winning pixel represents the object size; the number of winning pixels represents the number of objects. The use of an analog WTA circuit contributed to simple implementation. The sensor operated successfully for images in which the sizes of objects were equal to or smaller than $4 \times 4$ pixels.
\end{abstract}

\section{Keywords: smart sensor, Winner-Take-All circuit, transistor mismatch, CMOS imager}

\section{Introduction}

In order to speed up the process of pattern recognition, it is desirable to perform a preliminary level of image processing on-chip. In particular, detection of important features reduces a large amount of image data and hence facilitates higher level processing tasks carried out on a host computer. Both analog and digital approaches have been taken to realize on-chip processing. While analog sensors are usually implemented for specific purposes ${ }^{(1) \text {-(3) }}$, digital vision chips have programming flexibility to realize various kinds of processing ${ }^{(4)-(6)}$.

The on-chip function reported in this paper is to count the number of objects. This function is considered useful for applications such as cell counting, radar target detection, etc. Although this may be realized by using digital vision chips, no dedicated chip based on an analog design has been implemented so far. The analog approach makes implementation simpler, resulting in faster processing. We have successfully implemented an analog sensor for counting the number of objects by applying a Winner-TakeAll (WTA) operation to inherent mismatch in transistor characteristics. Furthermore, in the process of counting the number of objects, the sensor produces outputs which indicate the size of each object.

In the following, an algorithm based on the WTA scheme is described first. The circuit implementation is presented next with some simulation results. Finally, experimental results obtained using a fabricated sensor are presented.

\section{Algorithm}

Consider the binary image obtained by a sensor as shown in Fig.1. An object is defined in this paper as a set of mutually connected active (illuminated) pixels in either a horizontal or a vertical direction. Although it is easy for a human to count the number of objects, which is four in this example, it is a rather difficult task for a machine to carry out. One possible way of counting the number of objects is

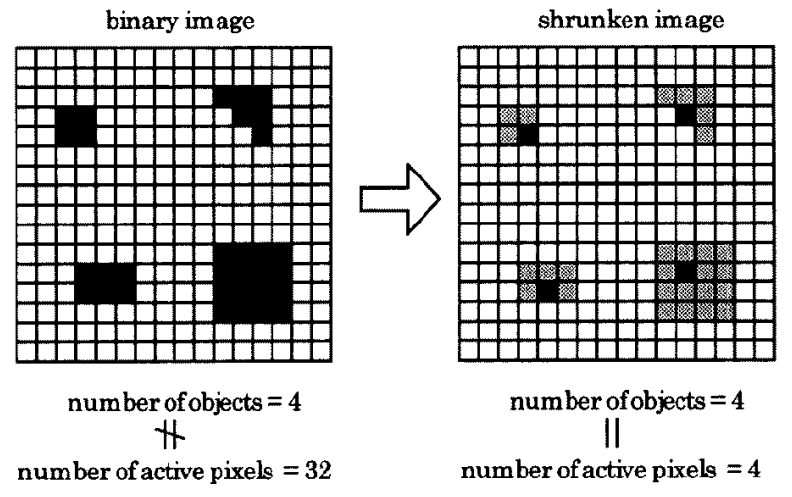

Fig.1 Method of counting the number of objects. The number of active pixels is different from the number of objects (left). After each object is shrunken to one pixel, the number of active pixels is the same as the number of objects (right).

to shrink each object to one pixel. Then by simply counting the number of active pixels, which can be easily implemented in a circuit, the number of objects is obtained. Fig.2(a) shows the shrinking procedure based on the cellular automaton model. Each pixel status is updated at every operation clock based on the status of itself and its neighbors. Although it is possible to eventually shrink any object to one pixel by properly specifying a rule for updating, the rule is not as simple as it first looks to cover all possible cases. Furthermore, because the processing is done in an iterative way, processing time is almost proportional to the size of the object.

In contrast to the above digital approach, an analog approach based on a Winner-Take-All (WTA) scheme is shown in Fig.2(b). Note that, in an analog sense, each active pixel has a different value. Within each object, the WTA scheme selects the pixel with the maximum value as a 


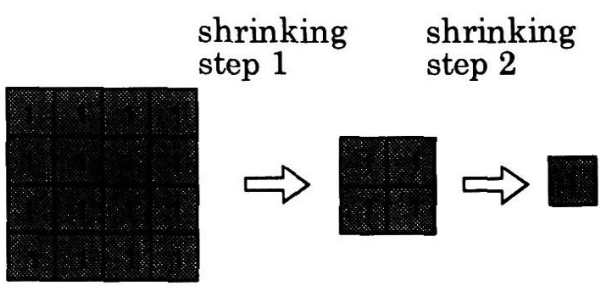

(a)

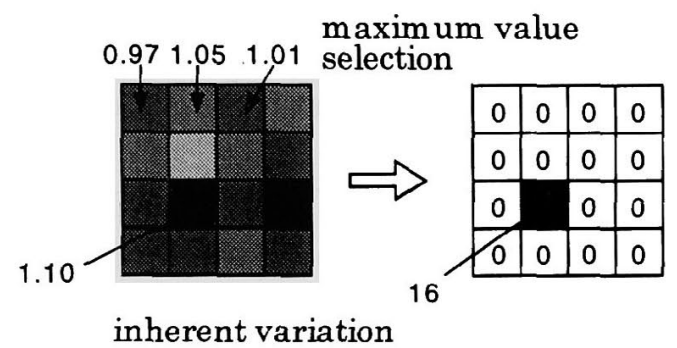

(b)

Fig.2 Digital and analog methods for number counting. (a) Shrinking procedure of an object to one pixel based on the cellular automaton model. (b) WTA scheme selects the pixel with the maximum value.

winner and suppresses the other pixels. Furthermore, the output of the winning pixel is proportional to the number of pixels and hence represents the size of the object.

Fig. 3 shows the measurement result of the variance in the current outputs of transistors. Thirty PMOS transistors, having the same gate dimension of $(w / l)=(3 \mu \mathrm{m} / 2 \mu \mathrm{m})$, are biased with the common voltage to produce a set of currents around $4 \mu \mathrm{A}$. The relative standard deviation was $5.5 \%$. These transistors were implemented using $2 \mu \mathrm{m}$ CMOS technology. The measurement result shows the importance of realizing a sharp WTA selectivity to be able to select only one winner.

\section{Implementation}

The circuit for the WTA operation is shown in Fig.4. Only five units connected in a one-dimensional fashion are shown for explanation purposes. The circuit, which is proposed by Lazzaro ${ }^{(7)}$, is used in the strong inversion region to increase its operational speed. For a set of input currents shown in the figure, the circuit selects unit 3 as a winner and suppresses the other units. The output current of unit 3 is equal to five times the reference current (5Iref), which represents the number of units.

The operation of the circuit is briefly explained below. The gate-source voltage $\mathrm{V}_{\mathrm{GSI}}(3)$ in unit 3 is determined by Iin(3), which is the largest input current. If the lateral resistance connecting adjacent units is small, voltages $V_{\mathrm{GS} 1}$ in the other units are very close to $\mathrm{V}_{\mathrm{GS} 1}(3)$. Therefore, the drain voltage of transistor $\mathbf{M}_{1}$ has to drop to accommodate the input current smaller than Iin(3), causing transistor $M_{2}$ to turn off. If transistors $M_{2}$ in the units other than unit 3 are turned off, all the reference currents (Iref) have to be

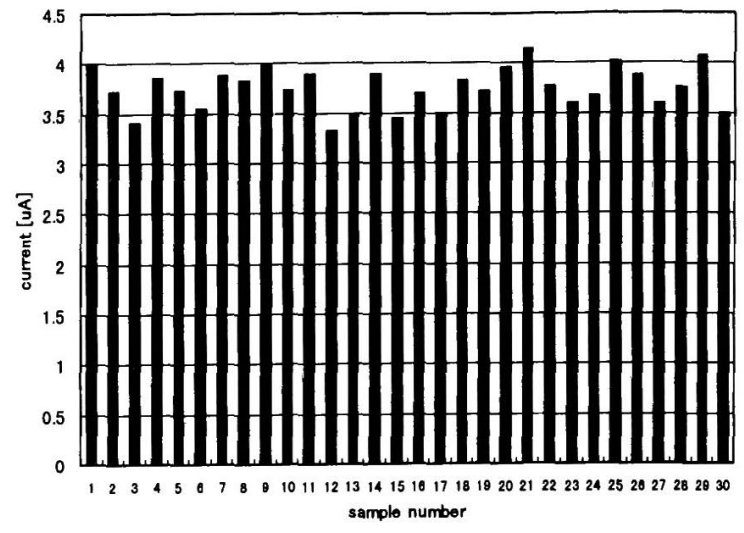

Fig.3 Variation of the output current from PMOS whose gate size is $3 \mu \mathrm{m} / 2 \mu \mathrm{m}$ in Orbit $2 \mu \mathrm{m}$ technology.
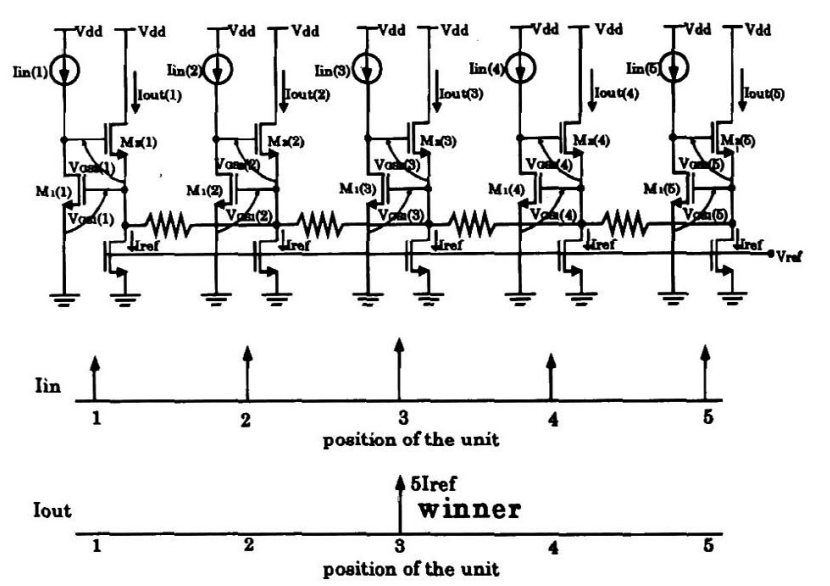

Fig.4 WTA circuit. For a set of input currents Iin, the winner-take-all circuit selects unit 3 as a winner and suppresses other units, resulting in the output current of SIref in unit 3.

supplied through $\mathrm{M}_{2}(3)$, resulting in an output current of 5Iref in unit 3. The lateral resistance controls the strength of suppression: more pixels are suppressed with a smaller resistance.

The WTA operation has to be carried out within each object. Fig.5 shows the circuit schematic explaining the method of forming an object. Each pixel has four switches for four directions. When a pixel is active (shown black in the figure), these four switches are closed. Therefore, two adjacent pixels which are both active are connected through two series NMOS switches. A set of active pixels belonging to the same object are thus mutually connected in either a horizontal or a vertical direction to electrically form an object. The formed object is separated from the surrounding inactive pixels.

Fig. 6 shows the schematic of the pixel implemented in the sensor. The pixel consists of a phototransistor and the WTA circuit. A photocurrent generated at a pnp 


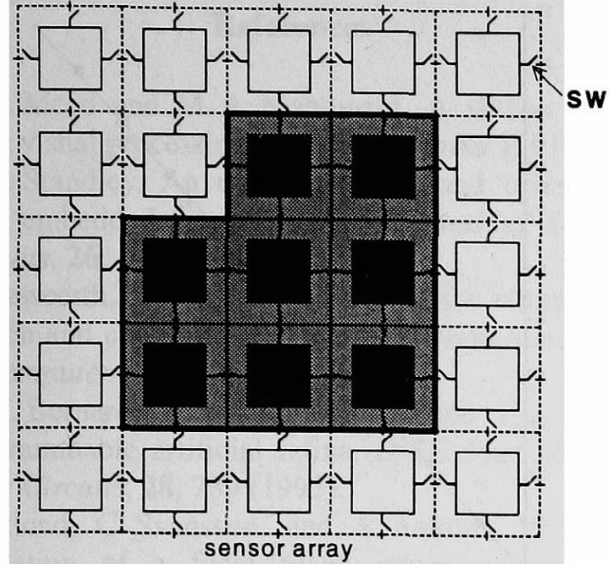

Fig.5 Method of forming an object. A set of black pixels in the center represents an object.

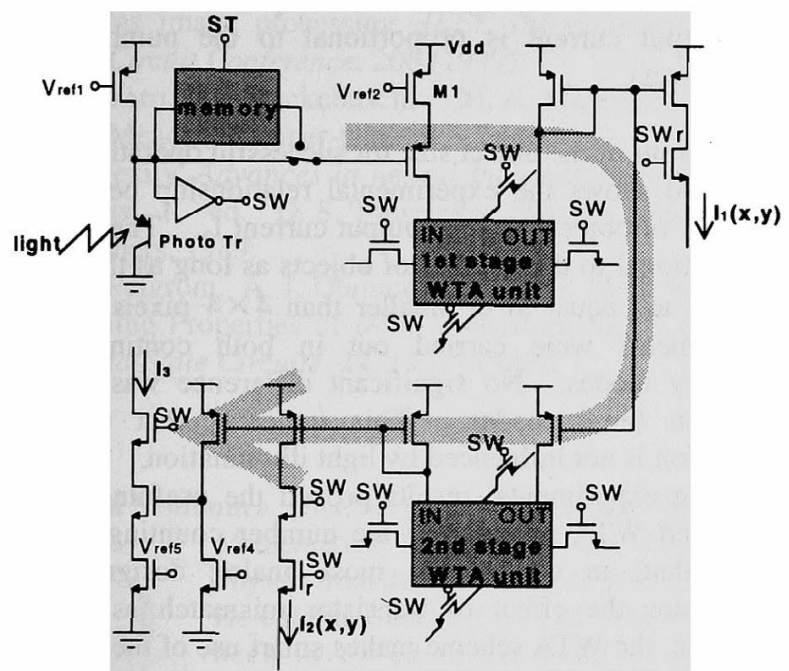

Fig.6 Schematic of a pixel. The WTA circuit is connected in a cascaded fashion. Pixels are connected by the switch in four directions.

phototransistor is compared to the threshold given by Vref1 for image digitization. A pixel under illumination is classified as active ( $\mathrm{SW}=\mathrm{Vdd}$ ) and allows the input current to flow into the WTA circuit. A pixel has NMOS switches in four directions, which are closed when the pixel is classified as active as explained in Fig.5. Thus, pixels belonging to the same object are connected with each other and isolated from the surrounding inactive pixels.

The resistance of the NMOS switch when closed corresponds to the lateral resistance illustrated in Fig.4. The input current to the WTA circuit, which is given by Vref2, has some variation due to the mismatch between transistors $M_{1}$ as shown in Fig.3. In order to realize large variation, the minimum size transistor, whose gate dimension is $3 \mu \mathrm{m} / 2 \mu \mathrm{m}$, is used to produce a set of current around $1 \mu \mathrm{A}^{(8)}$. The relative standard deviation of the current outputs of $1 \mu \mathrm{A}$ is expected to be larger than $5.5 \%$

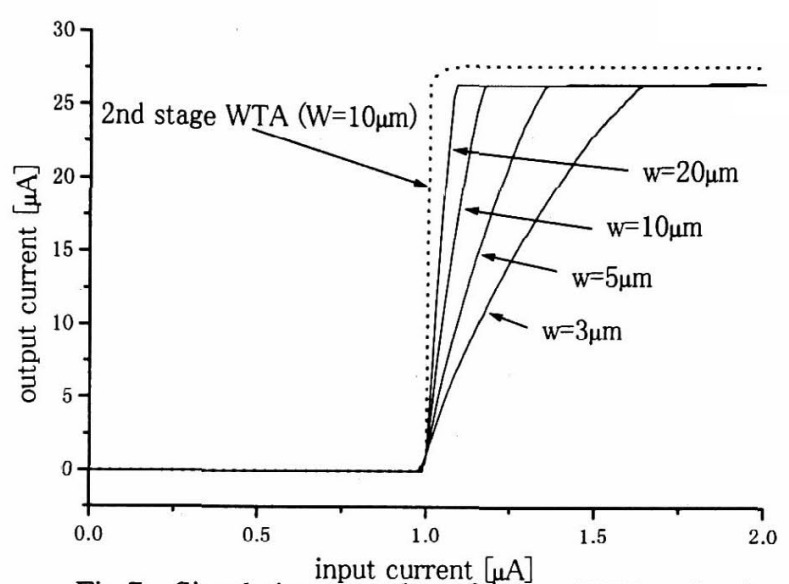

Fig.7 Simulation result of the WTA circuit connected in an array of $5 \times 5$.

obtained at the current level of $4 \mu \mathrm{A}$ (Fig.3).

The WTA circuit is configured in a cascaded form to enhance its winner selection capability: the second stage WTA circuit takes a mirrored output of the first stage WTA circuit, $I_{1}(x, y)$, as an input to produce an output current $I_{2}(x, y)$. The output current $I_{2}(x, y)$ at the winning pixel indicates the object size. Another current output, $\mathrm{I}_{3}$, which is the summation result of the reference current determined by Vref5 from winner nodes, is proportional to the number of nonzero $I_{2}(x, y)$ and hence represents the number of objects in an image.

In addition to the normal operation mode, in which the WTA operation is carried out for a continuously digitized image, the WTA operation can be performed for the result stored in the memory at one point in time. This operation mode, referred to as memory mode, makes it possible to carry out the WTA operation under dark conditions to eliminate the effect of light on the WTA performance.

Fig.7 shows one of the simulation results using the HSPICE circuit simulator. For a pixel array of $5 \times 5$, each having an input of $1 \mu \mathrm{A}$, the input at the center pixel is swept from 0 to $2 \mu \mathrm{A}$. Four solid lines show the output of the first stage WTA circuit at the center pixel for different values of gate width $(w)$ for the NMOS transistors used for connection.

The gate length $(l)$ is chosen as the minimal dimension of $2 \mu \mathrm{m}$. A smaller current is enough to become a winner for larger values of $w$. This is due to the reduced resistance between pixels. For $w=10 \mu \mathrm{m}$, a current of $1.15 \mu \mathrm{A}$ is required to be a full winner. The dotted line indicates the output current of the second stage WTA circuit for $w=10 \mu \mathrm{m}$. The selectivity is considerably improved: only a current of $1.01 \mu \mathrm{A}$ is necessary to become a full winner. Other simulation results showed that larger currents are necessary for the center pixel to become a winner for larger objects.

A prototype sensor was fabricated using a $2 \mu \mathrm{m}$ CMOS technology through MOSIS. Fig. 8 shows the photograph of the fabricated chip. The chip measures $6.8 \mathrm{~mm} \times 4.6 \mathrm{~mm}$ and has an array of $24 \times 14$ pixels. Each pixel measures 


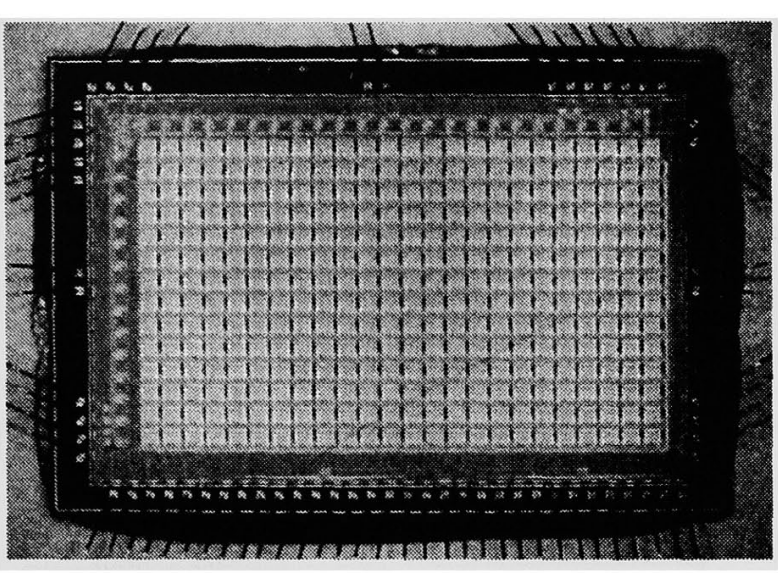

Fig.8 Photograph of the fabricated sensor.

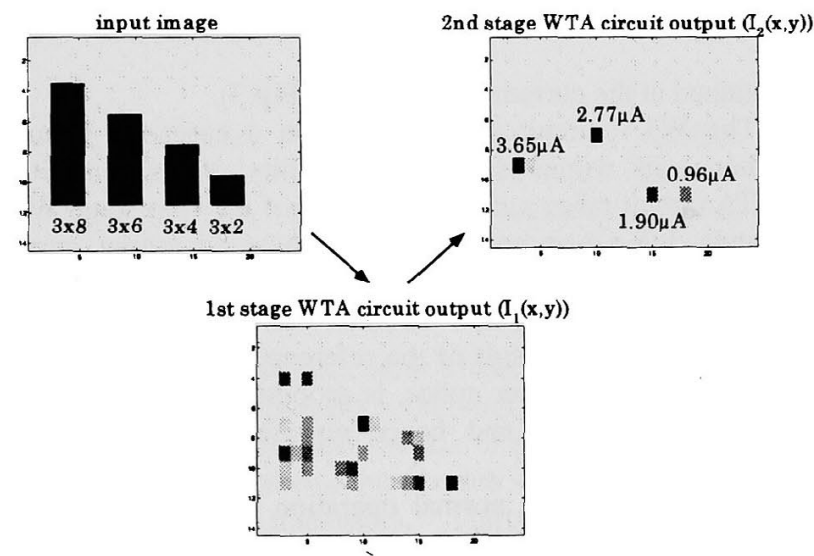

Fig.9 Experimental evaluation of the sensor performance. Only one pixel is selected as a winner from each object and the amount of current is proportional to the object size.

$250 \mu \mathrm{m} \times 250 \mu \mathrm{m}$ and contains a phototransistor of $121 \mu \mathrm{m} \times$ $27 \mu \mathrm{m}$. The phototransistor is implemented as a parasitic pnp structure with its base floating. Shift registers are placed in both $\mathrm{x}$ and $\mathrm{y}$ directions to sequentially read out the current outputs off chip to analyze the operation result.

\section{Experimental Results}

Various images were projected on the sensor for testing. Fig. 9 shows the responses of the WTA circuit, $I_{1}(x, y)$ and $I_{2}(x, y)$, for the image that has four rectangles of different sizes. Although suppression by the winning pixel is not strong enough to generate only one winner at the first stage, the second stage WTA circuit produced only one winner from each object. The amount of the current at the winning pixel is proportional to the size of the object. For square images, the WTA circuit is able to select one pixel from an object of $4 \times 4$ pixels or smaller. Objects of $5 \times 5$ pixels or larger sometimes produced multiple winners. A larger value of $w$ for the connection transistor should lift the

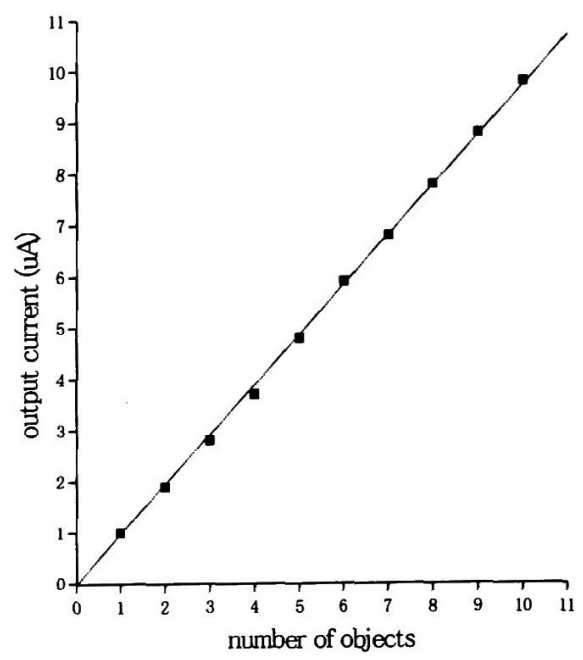

Fig.10 Experimental relationship between the number of objects and the output current. The output current is proportional to the number of objects.

upper limit of the object size for successful operation.

Fig.10 shows the experimental relationship between the number of objects and the output current $I_{3}$. The current is proportional to the number of objects as long as the sizes of objects are equal to or smaller than $4 \times 4$ pixels. These experiments were carried out in both continuous and memory modes. No significant difference was observed between these modes. This indicates that the WTA operation is not influenced by light illumination.

These experimental results proved the usefulness of the proposed WTA scheme for the number counting problem. Note that, in contrast to most analog designs, which eliminates the effect of transistor mismatch as much as possible, the WTA scheme makes smart use of the mismatch for simple implementation. Further extension of this research should include a detailed analysis on the relationship between the variation in a set of input currents and the performance of the WTA circuit for various shapes of objects.

\section{Conclusion}

The sensor that applies the WTA scheme to inherent transistor mismatch was designed and successfully tested to measure the number and the size of objects. The analog implementation based on the WTA scheme is simpler than digital implementation. To the knowledge of the authors, this is the first analog implementation of this kind.

\section{Acknowledgements}

The authors would like to thank Katsuyuki Sunamura at Sankyo Co., Ltd. for his technical assistance in circuit simulation and device testing.

(Manuscript received Oct. 4, 1999, revised Feb. 9, 2000) 


\section{References}

(1) C. A. Mead and M. A. Mahowald, A silicon model of early visual processing, Neural Networks,1, 91 (1991).

(2) D. L. Standley, An object position and orientation IC with embedded imager, IEEE Journal of Solid State Circuits, 26, 1853 (1991).

(3) S. Deweerth, Analog VLSI circuits for stimulus localization and centroid computation, International Journal of Computer Vision, 8, 191 (1992).

(4) T. M. Bernard, B. Y. Zavidovique, and F. J. Devos, A programmable artificial retina, IEEE Journal of SolidState Circuits, 28, 789 (1993).

(5) J. Eklund, C. Svensson, and A. Åström, VLSI implementation of a focal plane image processor - A realization of the near-sensor image processing chip concept, IEEE Trans. on VLSI Systems, 4, 322 (1996).

(6) M. Ishikawa, K. Ogawa, T. Komuro, and I. Ishii, A CMOS vision chip with SIMD processing element array for $1 \mathrm{~ms}$ image processing, IEEE International SolidState Circuit Conference, 206 (1999).

(7) J. Lazzaro, S. Ryckebusch, M. A. Mahowald, and C.A. Mead, Winner-take-all networks of $\mathrm{O}(\mathrm{N})$ complexity, Advances in neural information processing systems 1, ed. D.S. Touretzky Morgan Kaufmann Publishers, 1989.

(8) M. J. Pelgrom, A. J. Duinmaijer, and A. P. G. Welbers, Matching Properties of MOS transistors, IEEE Journal of Solid-State Circuits, 24, 1433 (1989).

Masatoshi Nishimura (member) received his B.S. degree in Mathematical Engineering and Information Physics from the University of Tokyo in 1984. He entered Sankyo Co., Ltd. where he had been involved in the field of medical engineering and currently in the field of bioinformatics. From 1991 to 1994, he was at the Department of Electrical Engineering, University of Pennsylvania. Since then he has been working on the design and implementation of smart optical sensors for pattern recognition.

Jan Van der Spiegel (non-member) received his engineering

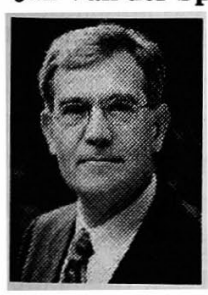
degree in Electro-Mechanical engineering and his Ph.D. degree in Electrical Engineering from the University of Leuven, Belgium in 1974 and 1979, respectively. He joined the University of Pennsylvania in 1980 where he is currently a professor of Electrical Engineering and Chairman of the Department. His research interests are in intelligent sensors, biologically inspired sensing systems, and analog and digital integrated circuits. 